\title{
Particle Simulation of Gas Expansion and Condensation in Supersonic Jets"
}

\author{
Quanhua Sun ${ }^{\dagger}$ and Iain D. Boyd ${ }^{\ddagger}$ \\ Department of Aerospace Engineering, University of Michigan, Ann Arbor, MI 48109 \\ Kenneth E. Tatum ${ }^{* *}$ \\ Aerospace Testing Alliance, Arnold Air Force Base, TN 37389
}

\begin{abstract}
The direct simulation Monte Carlo (DSMC) method is used to simulate the flow created prior to formation of a Z-pinch in the Nuclear Weapons Effects (NWE) Decade facility at the Arnold Engineering Development Center (AEDC). The simulation domain and the inflow boundary conditions are determined on the basis of a continuum-based Computational Fluid Dynamics (CFD) analysis. The DSMC simulation shows that the rapid expansion of the argon gas in the dual shell, concentric annular nozzle leads to large supersaturated regions. A homogeneous condensation model is then proposed for the DSMC simulation to include the effects of the nucleation and condensation, which explains the principal differences between the simulation results and available experimental data.
\end{abstract}

\section{Introduction}

T he Decade Radiation Test Facility (DRTF), located at the U.S. Air Force's Arnold Engineering Development Center (AEDC), is a new advanced-generation radiation test facility designed to test $21^{\text {st }}$-century space and missile systems and their components. The DRTF is a world-class, unique facility producing multiple radiation environments to test various systems such as satellites and missile guidance controls and their components. A smaller X-ray simulator, the Modular Bremsstrahlung Source (MBS) provides nuclear weapons effects (NWE) testing on cables and small satellite components. It is believed that the mass flow distribution created prior to forming a Z-pinch in the Decade facility is critical in determining the resulting radiation effects. Physically accurate numerical simulations are expected to play a significant role in optimizing the performance of the facility and in enhancing the testing capabilities provided by AEDC.

The flow in the Decade Quad (DQ) is established through a gas-puff nozzle, which employs a dual shell, concentric annular geometry with an outer exit plane diameter of $12 \mathrm{~cm}$. The pinch region is about $4 \mathrm{~cm}$ in length. For a typical test run on the DQ, the plenum pressure is approximately 250 torr, and the vacuum chamber is set at $10^{-6}$ torr. The flow starts from room temperature with a flow rate of $3 \mathrm{~g} / \mathrm{sec}$. The argon density in the plume varies from about $10^{18} \mathrm{~m}^{-3}$ to $10^{23} \mathrm{~m}^{-3}$. The flow is transient for about $1 \mathrm{msec}$ before reaching steady-state conditions. These conditions result in a mean free path that indicates the gas expansion is in the kinetic flow regime. Therefore, correct simulation of the flow requires a kinetic approach, such as the direct simulation Monte Carlo (DSMC) method. ${ }^{1}$

Current computational resources, however, indicate that the Decade flow is too dense for the DSMC method alone to simulate the entire flow. A continuum-based computational fluid dynamics (CFD) analysis can indicate regions where non-continuum nonequilibrium effects are important through the calculation of empirical continuum breakdown parameters. A continuum-based solution also provides necessary inflow boundary conditions for a DSMC simulation to use.

\footnotetext{
${ }^{*}$ The research reported herein was performed by the Arnold Engineering Development Center (AEDC), Air Force Materiel Command. Work and analysis for this research were performed by personnel of the University of Michigan and by personnel of Aerospace Testing Alliance, the operations, maintenance, information management, and support contractor for AEDC. Further reproduction is authorized to satisfy needs of the U. S. Government.

${ }^{\dagger}$ Post-Doctoral Research Fellow, Member AIAA.

*Professor, Associate Fellow AIAA.

${ }^{* *}$ Engineer/Scientist VI, IT\&E Dept., Sr. Member AIAA.
} 
Another challenge in simulating the Decade flow is the possible nucleation and condensation of the argon gas during the rapid expansion process. In the absence of foreign nuclei, argon clusters can form if the flow has a large degree of supersaturation. After clusters reach the critical size, homogeneous condensation begins, which transfers mass from, and adds energy to, the gas phase--thus significantly changing the flow field.

In the literature, prior studies on homogeneous nucleation and condensation focused primarily on the nucleation rate and cluster growth. ${ }^{2-9}$ Few studies have been reported of the cluster effects on flow field. ${ }^{10-12}$ For instance, Zhong et al. ${ }^{13}$ studied homogeneous condensation in supersonic plumes using the DSMC method without tackling the impact on the vapor phase. One exception is the work of Perrell et al., ${ }^{14}$ who developed a continuum-based approach for calculating supersonic, two-dimensional nozzle flows, including "heating" caused by the phase change although the continuum-based approach is questionable as a method to correctly predict the flow field.

In this paper, the Decade flow is simulated using both the continuum-based approach and the DSMC method. The CFD and DSMC simulations are described in Sections 2 and 3, respectively. Section 4 introduces a homogeneous condensation model suitable for the DSMC method, and simulations, including this model, are discussed in Section 5. Finally, some concluding remarks are given in Section 6.

\section{Continuum-Based Simulation}

The nozzle used in this investigation was designed by Alameda Applied Sciences Corporation. It is composed of two internal concentric annular nozzles and a recess, as sketched in Fig. 1. The internal nozzles accelerate and expand the argon gas from the plenum, and the resulting two supersonic ring jets expand further into the recess and the vacuum chamber (pinch area). The nozzles and the vacuum chamber comprise the entire simulation domain.

The commercial computational fluid dynamics software $\mathrm{GASP}^{15}$ is used to simulate the entire flow field, which is divided into 19 computational zones having a total of 179,400 cells. Within the GASP software, the turbulence model used is Menter's shear stress transport (SST) model, a $k-\omega$-like model that attempts to blend the features of Wilcox's $k-\omega$ model with the $k-\varepsilon$ model to improve free shear layer modeling. Both viscosity and thermal conductivity are calculated using Sutherland's law. The fluxes are calculated using Roe's third-order fluxdifferences, where the min-mod limiter is employed to limit forward and backward gradients of the primitive

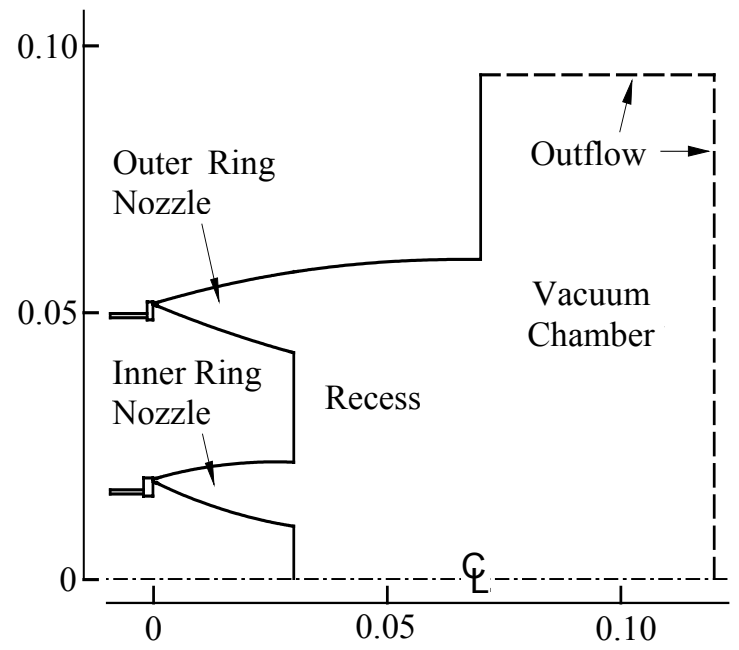

Figure 1. Computational Domain for the Decade flow variables in the vicinity of discontinuities for the reconstruction of left and right states. The equations are integrated to the steady state using a Gauss-Seidel implicit scheme with Gauss-Seidel inner iterations at each pseudo time step. Local time stepping is employed to accelerate convergence, and the CFL number is ramped from a minimum to a maximum for each grid sequence. Three grid sequences are used.

To establish the flow from the plenum to supersonic outflow boundaries, the actual solution is performed in stages. First the inner and outer ring internal nozzle flows are computed independently, initially with low (nearvacuum) back pressure downstream boundary conditions; then the flows are transitioned to extrapolation outflow boundary conditions (using grid sequencing). The outflow results are extracted and specified as inflow boundary conditions to compute a recess and vacuum chamber flow field, with low back pressure downstream boundary conditions (again employing grid sequencing). Finally, the three preliminary solutions are extracted and used to initialize a fine grid of the entire flow field; additional iterations are performed to blend the pieces, smooth out inconsistencies, and further converge the results. Extrapolation outflow boundary conditions are specified at this step.

The CFD simulation shows that the Decade flow is very complex, as illustrated by the density flow-field plot, Fig. 2. The density of argon gas drops rapidly from the plenum to the internal ring nozzles as a result of the expansion. Strong expansion is also observed in the outer part of the vacuum chamber, where the density is extremely low. The low density indicates that rarefaction effects may be important in certain flow regions. To identify these regions, two continuum breakdown parameters are used to evaluate the flow. One parameter is Bird's continuum breakdown parameter, $P,{ }^{1}$ and the other is the gradient-length local Knudsen number, $K n_{G L L}::^{16}$ 


$$
K n_{G L L}=\max \left(\frac{\lambda}{\rho} \nabla \rho, \frac{\lambda}{T} \nabla T\right)
$$

where $\lambda$ is the mean free path of gas molecules, $\rho$ is the density, and $T$ is the temperature. Bird's parameter, $P$, and the local Knudsen number display similar trends for the flow field; the local Knudsen number contours are plotted in Fig. 3. Generally, non-continuum nonequilibrium effects are expected to become important when the local Knudsen number exceeds 0.05 . This means that nonequilibrium is expected around the corners between the recess and the internal nozzles, and in the outer part of the vacuum chamber. Therefore, the DSMC method is needed to simulate the flow in the recess and the vacuum chamber. The continuum-based results, however, offer inflow boundary conditions and initial conditions for the DSMC simulation.

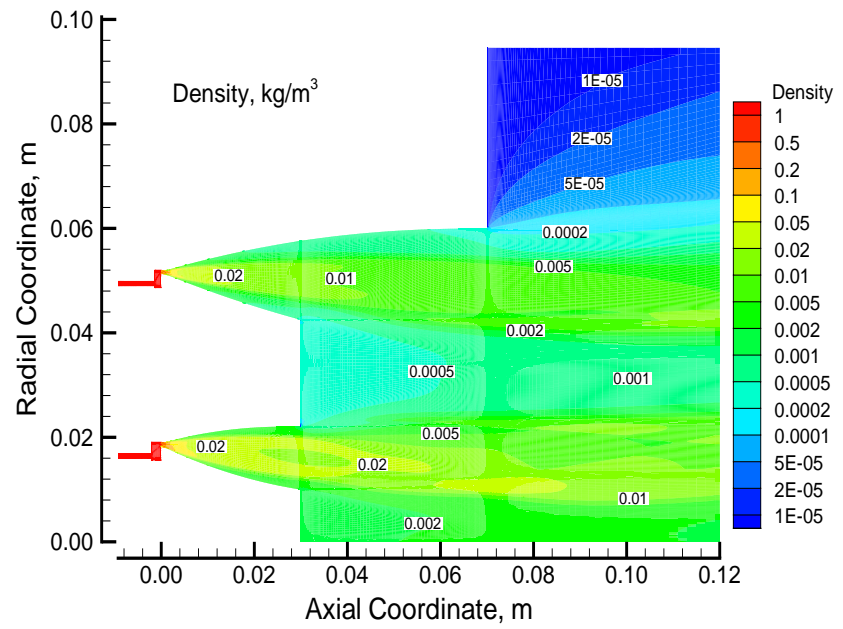

Figure 2. Density Flow Field Predicted by the CFD Simulation

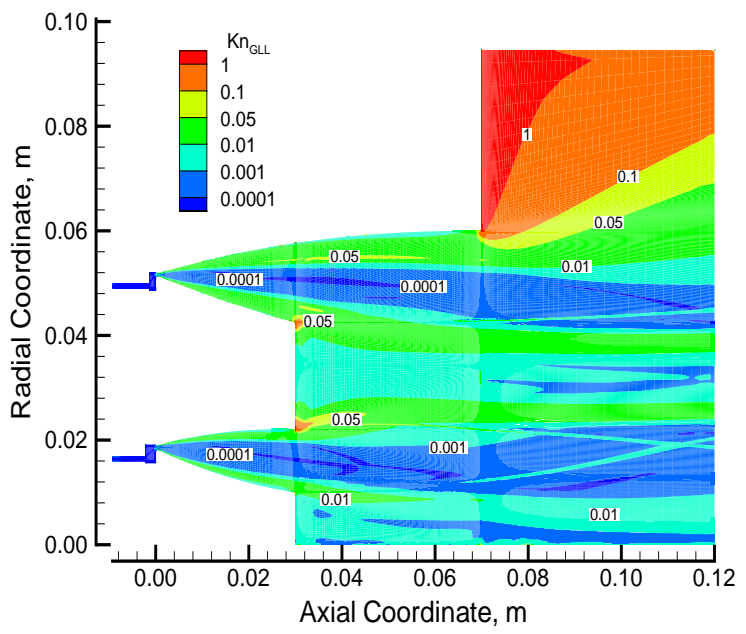

Figure 3. Contours of the Gradient-Length Local Knudsen Number Based on the CFD Simulation

\section{Particle Simulation}

The direct simulation Monte Carlo (DSMC) method $^{1}$ is a mature approach for modeling rarefied flows, such as those occurring in the Decade facility. The DSMC method simulates gas flows through computations of motions and collisions of modeled particles, each of which represents a large number of real physical atoms or molecules. The primary approximation is to uncouple the molecular motions and intermolecular collisions over small time intervals, where particle motions are modeled deterministically while the collisions are treated statistically. The DSMC method is applied to the flow in the recess and vacuum chamber only because the continuum-based CFD approach is numerically less expensive and is valid to predict the flow within the internal ring nozzles.

The flow is simulated using the state-of-the-art DSMC research code MONACO. ${ }^{17}$ The variable hard sphere (VHS) molecular model is specified in this case, which assumes that the total collision cross section depends on the relative collision velocity. The computational domain (recess and vacuum chamber) for the DSMC simulation is divided into 794,448 structured cells. These cells are carefully designed to control the total number of cells. The cell size in the radial direction is approximately one mean free path of the argon gas, where there are obvious flow gradients. The cell size in the axial direction is much larger, but less than 10 times the mean free path of the molecules where the flow gradients are small. However, the DSMC method generally requires a cell size less than the mean free path of molecules. The validity of the solution due to the larger cell size is examined using the sub-cell technique where particles are selected for collisions within a smaller distance. No obvious difference is detected between solutions, even when nine sub-cells are employed within each cell. Therefore, the designed mesh is considered acceptable for this investigation.

A particle weight, the number of real atoms represented by one simulated particle, is used for each computational cell so that a similar number of particles are simulated in each cell. In the simulation, roughly 20 particles are employed in each cell, and the total number of simulated particles is about 16 million. The inflow boundary conditions are extrapolated from the continuum-based results. The outflow boundary conditions are self- 
extrapolated from simulated results and several iterations are performed. The time step is $5 \times 10^{-9} \mathrm{sec}$, which is less than the mean collision time of molecules, and more than 200,000 time steps are simulated. To ensure that the simulation reaches a steady state, two different initial conditions are employed. One is a vacuum condition, and the second is extrapolated from the continuum-based results. A good steady state is assumed to be reached when the results using the two initial conditions become indistinguishable.

The DSMC simulation is very expensive, and it requires approximately two days running on a 20 processor Linux cluster for one case. The simulation results are shown in Figs. 4 through 6 . The results illustrate the complexity of fluid interaction that occurs as the two ring jets expand and mix. The density distribution shown in Fig. 4 is similar to that predicted by the continuum-based approach. The results indicate that the two ring jets expand very little as they travel through the recess and the vacuum chamber. This is because the hot gas between the jets blocks the expansion of the cold jets. The temperature distribution is shown in Fig. 5, which indicates that the ring jets are very cold and that there are regions where the gas temperature is far below $30 \mathrm{~K}$. The low temperature also means that the Mach number of the flow is large, as shown in Fig. 6. The presence of supersonic outflow implies that outflow boundary conditions are not critical to the simulation.

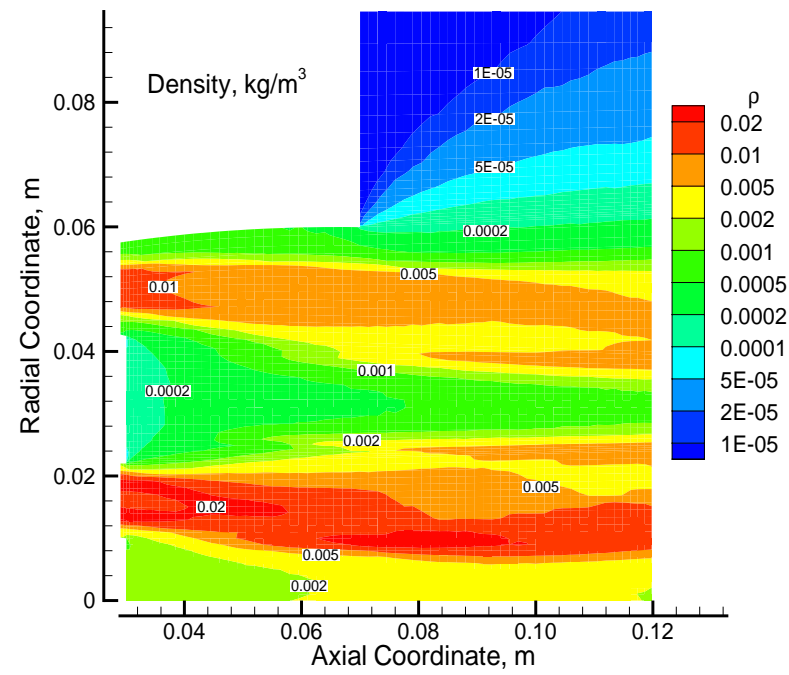

Figure 4. Density Flow Field Predicted by the DSMC Simulation
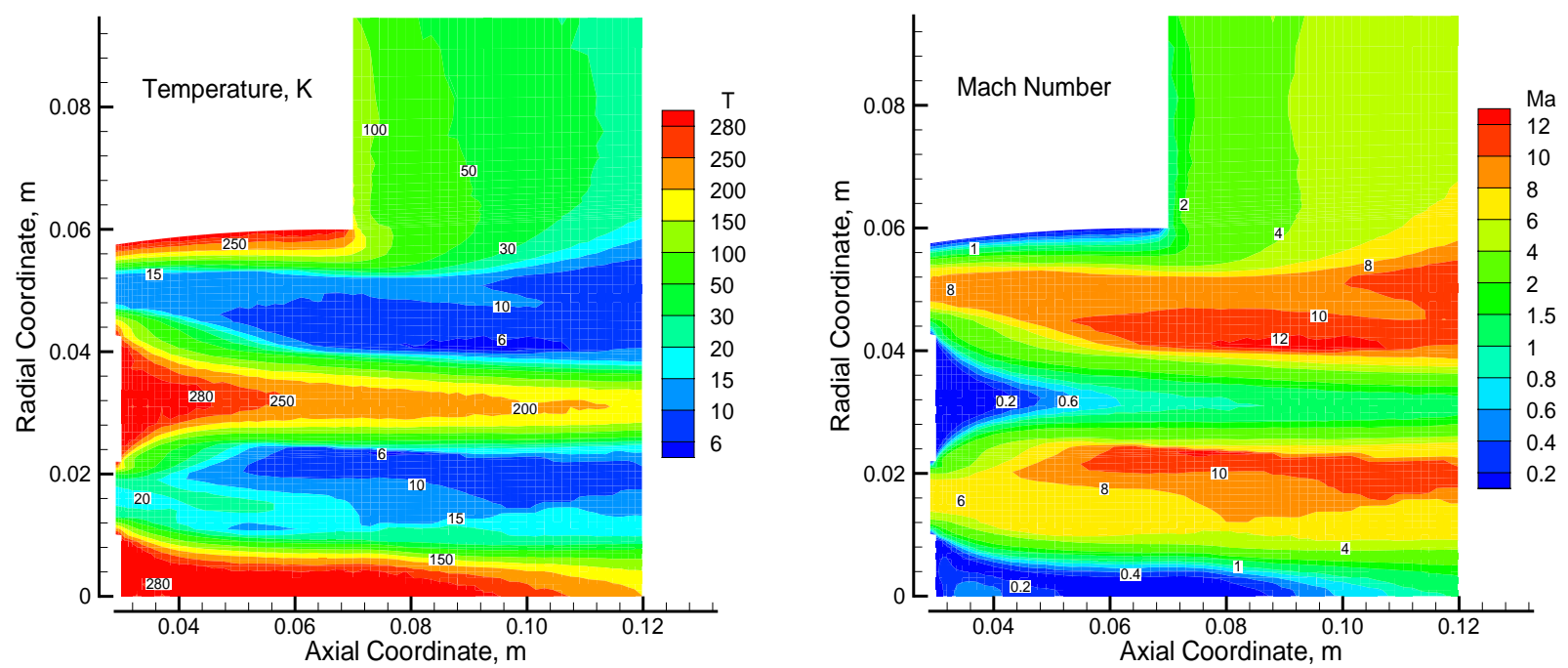

Figure 5. Temperature Distribution Predicted by the DSMC Simulation

Figure 6. Mach Number Distribution Predicted by the DSMC Simulation

It is well known that nucleation and condensation will occur when the temperature is sufficiently low; this condition is characterized by the degree of supersaturation, $S$ : the ratio of the gas pressure to the vapor pressure $\left(P_{s}\right)$ at the same temperature. The vapor pressure of argon gas at low temperature can be approximated using a logarithmic-exponential curve-fit expression as follows:

$$
\ln P_{s}=-396.465+225.279 \ln T-41.7722 \ln ^{2} T+2.6374 \ln ^{3} T
$$

when the temperature is in the range between $20 \mathrm{~K}$ and $150.85 \mathrm{~K}$ (the critical temperature). Using this expression, the degree of supersaturation for the simulated flow was computed and is shown in Fig. 7. Clearly, the ring jets are highly supersaturated, which means homogeneous nucleation and condensation are expected to occur before the gas temperature decreases to very low values. It should be noted that nucleation is very likely to start from within the 
internal ring nozzle regions, and that an enhanced continuum-based simulation using a condensation model will give better inflow boundary conditions for the DSMC simulation.

It is important to compare simulation results with experimental data. The only experimental data available are radial density profiles as determined by laser interferometry measurements at several locations (private communication with Alameda Applied Sciences Corporation). These data are plotted in Figs. 8 and 9 at axial locations of $0.072 \mathrm{~m}$ and $0.1 \mathrm{~m}$, respectively, as measured from the throat. The error bars indicate the estimated measurement error, and the simulation curves are interpolated from the CFD and DSMC results. The CFD and DSMC results agree well, but large differences are observed between the simulation results and the measured data. The experimental data show that the two ring jets expand more widely, as compared to the simulation results, and mix when they approach each other. This behavior is

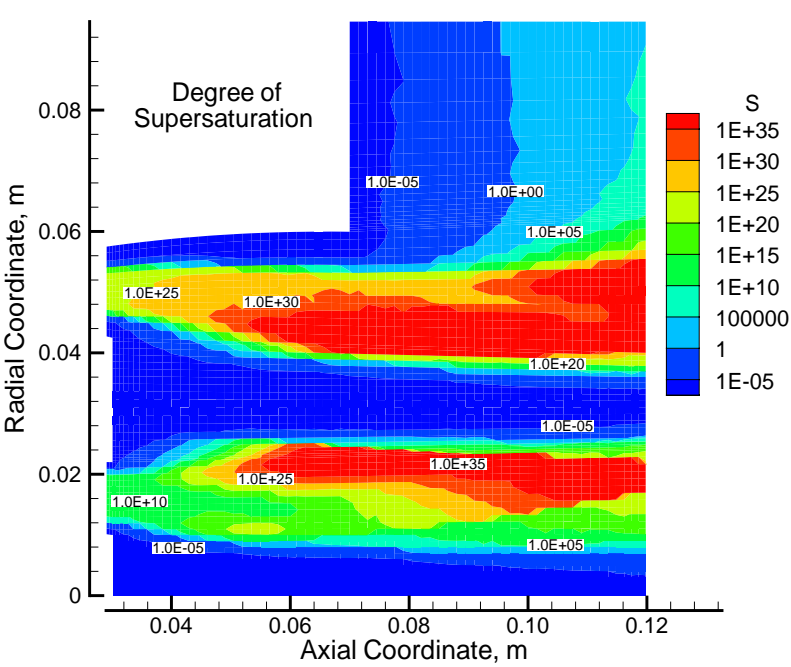

Figure 7. Degree of Supersaturation Computed Based on the DSMC Simulation Results

consistent with possible condensation effects caused by the low temperature. The low temperature of the jets from the simulations results in low pressure and thus prevents the jets from expanding into nearby high-temperature regions. Also, the measured data have a density maximum closer to the centerline, possibly partially as a result of radial effects. The inner ring jet has more space for expansion outward (because the radius is larger) than inward, towards the axis. Thus, the mass density decreases quickly at larger radii while changes are small near the centerline, even as the jet expands further. Condensation effects will increase the expansion because of the temperature increase and therefore will shift the density maximum towards the centerline.

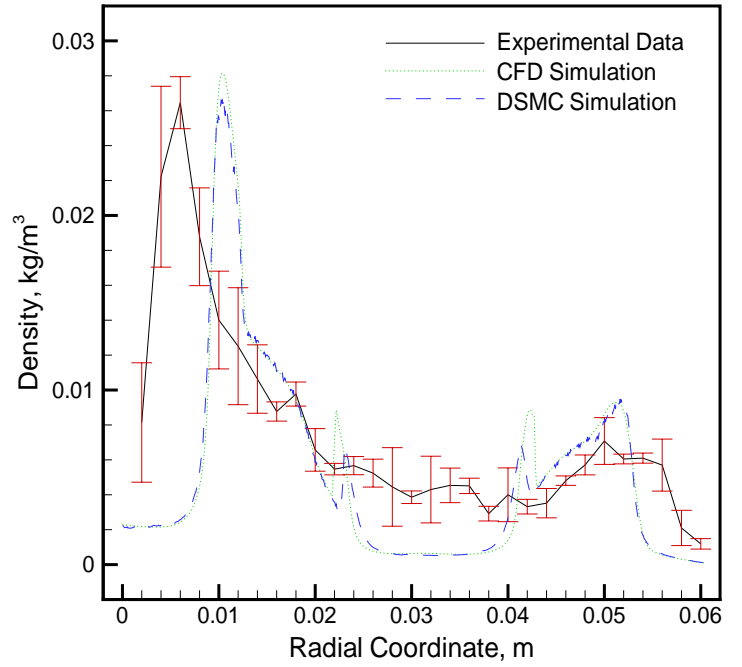

Figure 8. Density Profiles at the Axial Location of $0.072 \mathrm{~m}$

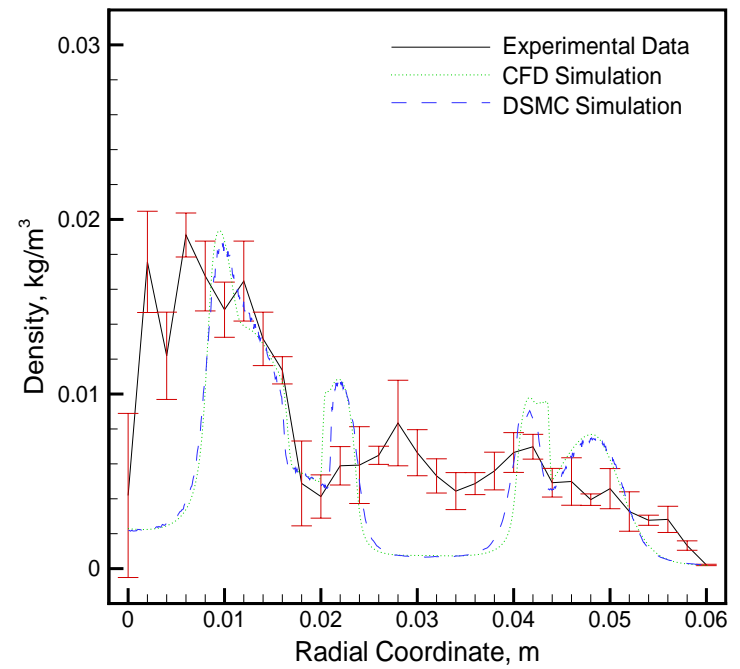

Figure 9. Density Profiles at the Axial Location of $0.1 \mathrm{~m}$

\section{Condensation Model}

\section{A. Homogeneous Nucleation Theory}

A gas is regarded as undersaturated, saturated, or supersaturated if the pressure of the gas is less than, equal to, or larger than the vapor pressure of the liquid phase at the same temperature. The undersaturated and saturated states are thermodynamically stable, while the supersaturated state is unstable. The supersaturated gas will nucleate and condense in the presence of impurities or walls. Otherwise, the gas sustains a very high degree of saturation until homogeneous nucleation occurs. 
Homogeneous nucleation occurs when the free energy loss from the transition of gas molecules into the liquid phase can compensate for the energy increase resulting from the surface tension (the energy barrier of classical nucleation theory). ${ }^{3}$ The energy loss caused by the phase change results from the van der Waals forces that bind molecules as tightly as in the liquid or solid state. The forces, however, have a limited effective range, and thus a bound molecule has interactions only with nearby molecules. This implies that a molecule near the surface of a droplet experiences surface effects that molecules away from the surface do not. Therefore, from the macroscopic viewpoint, a cluster or a crystal can form and expand as long as the energy released as a result of the phase change (volume dependent) is able to overcome the surface free energy (surface dependent).

Classical nucleation theory ${ }^{2}$ derives the nucleation rate on the basis of the balance between condensation and evaporation. The fundamental quantity is the free energy for cluster formation. Becker and Doring obtained an expression using a steady-state assumption, while Lothe and Pound obtained a similar expression that differs by a factor of $10^{17}$ when the free translation and rotation in the Helmholtz potential are considered. ${ }^{2}$ Surprisingly, on the basis of available experimental data, it is believed that some gases follow the Becker-Doring theory, while others follow the Lothe-Pound theory. It is generally believed that the Lothe-Pound theory is more accurate, although it fails for some gases with polar bias because such effects are not included.

Molecular approaches ${ }^{6-8}$ are also used to calculate the nucleation rate, the equilibrium cluster distribution, and the free energy. Because clusters can dissociate, the cluster distribution can diverge as the volume containing the clusters increases. Treatment of the volume dependence of this free energy has been a source of confusion and controversy in the development of a general molecular theory of nucleation.

There is also disagreement for modeling of the effects of surface tension (surface free energy). Some studies show that the surface tension decreases as the cluster size decreases, while other studies support the opposite trend. The binding energy is also size dependent. If the cluster size is infinite, the binding energy can reach the latent heat of condensation. If the cluster size is very small, the binding energy is negligible because very few degrees of freedom exist. Even for the same cluster size, there are many possible cluster configurations with different free energy possibilities. Hence, the nucleation process is not yet fully understood.

Therefore, to account for the cluster effects on a flow field, very general expressions for the cluster nucleation and condensation to be used in the DSMC simulation are proposed. The coefficients in the expressions are adjustable.

\section{B. Nucleation Rate}

According to classical nucleation theory, the rate of formation of new clusters, $J$, is given by:

$$
J=\sqrt{\frac{2 \sigma}{\pi m^{3}}} \frac{\rho_{v}^{2}}{\rho_{l}} \exp \left(-\frac{4 \pi r_{*}^{2} \sigma}{3 k T}\right)
$$

where $\sigma$ is the surface tension of the liquid, $m$ is the mass of gas molecules, $\rho_{v}$ is the vapor density, $\rho_{l}$ is the liquid density, $k$ is the Boltzmann constant, $T$ is the temperature, and $S$ is the degree of supersaturation. The radius of the critical-sized cluster, $r_{*}$, is given by:

$$
r_{*}=\frac{2 \sigma}{\rho_{l} R T \ln S}
$$

where $R$ is the ideal gas constant. The surface tension is temperature dependent, approximated by $0.0344\left(1-T / T_{c}\right)$ $\mathrm{N} / \mathrm{m}$ following the approach of Hale ${ }^{18}$ for argon gas, whose critical temperature, $T_{c}$, is about $150.85 \mathrm{~K}$.

The rate of formation of a new cluster can be expressed as the probability of nucleation of a collision particle pair, which can be implemented in the DSMC method. In the DSMC method, the collision rate is computed as:

$$
N_{c}=\frac{1}{2} \frac{\rho^{2}}{m^{2}} \overline{\sigma_{T} C_{r}}
$$

where $\sigma_{T}$ is the collision cross section, and $C_{r}$ is the relative velocity of the colliding particles. It can be shown that the probability for a collision pair to have nucleation is as follows:

$$
P_{N}=\frac{C_{1} \sqrt{8 m \sigma / \pi}}{\rho_{l}\left(\sigma_{T} C_{r}\right)} \exp \left(-\frac{4 \pi r_{*}^{2} \sigma}{3 k T}\right)
$$


where the constant $C_{1}$ is the number of molecules in the critical-sized cluster.

This nucleation probability is very small even when the degree of supersaturation is very high, requiring a large number of collisions to create one nucleate. For instance, the nucleation probability is on the order of $10^{-13}$ when the argon gas temperature is $50 \mathrm{~K}$ and the number density is $5 \times 10^{25}$ particles $/ \mathrm{m}^{3}$ (the degree of supersaturation is about $1,000)$. Many nucleation collisions can occur because the collision rate is very large $\left(\sim 10^{35} / \mathrm{m}^{3} / \mathrm{sec}\right)$. However, in the DSMC approach, simulated collisions are very limited, and it is rare to have one nucleate. The nucleation probability is multiplied by a factor of $10^{8}$ for current simulations in order for nucleation to occur. This approach may be questionable, but it can be a good choice for the Decade flow for the following reasons. First, one simulated particle represents a large number $\left(>10^{9}\right)$ of real molecules; second, nucleation occurs beginning in the internal nozzle regions; third, nonequilibrium nucleation is different from classical nucleation theory; and fourth, the condensation process dominates the cluster effects on the flow field.

\section{Condensation and Evaporation Rates}

The homogeneous nucleation rate is generally very small, and the phase change in supersonic expansion flows results primarily from the condensation process. Classical nucleation theory assumes the condensation rate can be approximated as the product of the sticking coefficient and the collision rate between monomers (single atoms) and clusters. The sticking coefficient, however, is very difficult to determine. On the other hand, a molecule collision can separate a monomer from a cluster, which represents evaporation. The evaporation rate can be similarly approximated as the product of an escaping coefficient and the collision rate between monomers and clusters. There are other types of condensation and evaporation, such as condensation caused by cluster-cluster collision and selfevaporation of clusters. However, collisions between monomers and clusters are the most likely occurrence because the population of monomers is the largest, according to the Boltzmann relation.

It is well known that collisions lead a flow towards an equilibrium state. Thus, condensation is more likely to occur than evaporation when the flow is supersaturated, and vice versa. Therefore, the probability for a monomercluster collision will produce condensation or evaporation can be reasonably expressed as:

$$
p_{c}=q_{c}(S-1)
$$

where $q_{c}$ is the coefficient of condensation (or evaporation - they are assumed to be equal), and $S$ is the ratio of the gas pressure to the vapor pressure measured at the same temperature. A positive value of $p_{c}$ indicates condensation, and a negative value refers to evaporation. This expression is consistent with the droplet growth equation used in Perrell et al. ${ }^{14}$

\section{Energy Related to Phase Change}

As discussed in Section 4.1, a certain amount of energy is released during nucleation or condensation. The amount of energy, however, is very difficult to determine. The latent heat of condensation can heat a volume of argon gas about 500 degrees Kelvin while the energy released during the formation of a dimer is negligible. A dimer is formed when two monomers stick together during a nucleation collision.

Apart from determining the magnitude of the unknown released energy, difficulties also exist in manipulating this energy. Physically, this energy is added to, or provided by, the collision pair or other collision partners if multiple particles are involved. However, it is very rare for particles to provide a sufficient amount of energy for an evaporation process during particle simulations. A simulation alternative is to share this energy among all the particles in the computational cell. This will, of course, speed up the energy relaxation, but it may be acceptable because the existence of clusters means that the density is relative large and there is a considerable number of collisions.

\section{DSMC Simulation with Condensation Model}

The purpose of the Decade flow simulation is to understand the flow field experienced in the Decade facility. This objective does not require investigating the cluster size distribution in the flow field. This would be a very difficult task because one simulated particle represents a large number of real molecules, and only a very limited number of particles are simulated due to limitations of computer resources. The most important effect of nucleation and condensation in the Decade flow is the addition of energy resulting from the phase change, which increases the gas temperature and affects the density distribution.

In this investigation, the dimer is the only argon cluster considered. When a monomer is supposed to attach to a dimer, a pair of monomers is selected according to a 50-percent possibility to form a dimer instead of a polymer. 
The dimer can be regarded as a big molecule with internal degrees of freedom; the internal energy is obtained when the dimer is formed and removed when the dimer is evaporated. However, the energy is not constant because the internal energy changes during particle collisions. An alternative is to neglect the internal energy of the dimer and consider the energy related to the phase change to be shared by all the particles in the computational cell. This shared energy is manipulated through particle collisions where the collision energy is adjusted.

A challenge to implementing the proposed nucleation and condensation model in the DSMC method is the evaluation of the temperature. A correct method of evaluation, offered by Sun and Boyd (unpublished), is to account for the effect of the limited sample size as follows:

$$
T_{0}=\frac{N_{0}}{N_{0}-1} \frac{m}{3 k}\left(\left\langle V_{1}^{2}+V_{2}^{2}+V_{3}^{2}\right\rangle-\left\langle V_{1}\right\rangle^{2}-\left\langle V_{2}\right\rangle^{2}-\left\langle V_{3}\right\rangle^{2}\right)
$$

where $N_{0}$ is the average number of particles in the cell, $V_{i}$ are velocity components, and \langle\rangle is the average over all the particles. A statistical analysis (Sun and Boyd) shows that the statistical scatter for the temperature evaluation is as follows:

$$
E_{T}==\sqrt{\frac{2 T_{0}^{2}}{3 N_{0}}}
$$

which is very large when the sample size is small. The scatter can be reduced using a sub-relaxation technique by including the previous history:

$$
\overline{T_{j}}=(1-\theta) \overline{T_{j-1}}+\theta T_{j}
$$

where $\theta$ is the sub-relaxation factor. It is found that the scatter can be reduced by a factor of $\sqrt{1 / \theta}$ that is greater than 30 when $\theta$ is set to 0.001 if the samples are statistically independent. The sub-relaxation technique is implemented so that the calculated temperature can be used for the nucleation and condensation model.

Simulations using the condensation model are performed where the phase change energy is tested. The condensation--evaporation coefficient $q_{c}$ in Eq. (7) is currently set to 0.1. Simulations show that the cluster formation changes the flow significantly. Figures 10 through 12 show the results when 30 percent of the latent energy of condensation is specified as the phase-change energy. The total mass density shown in Fig. 10 illustrates that the two ring jets expand more widely, compared to Fig. 4, as expected. The cluster mass density field, Fig. 11, shows that a considerable part of argon gas has been converted to the cluster state. The temperature is therefore increased considerably, as shown in Fig. 12. These results demonstrate the impact of gas condensation on the flow field.

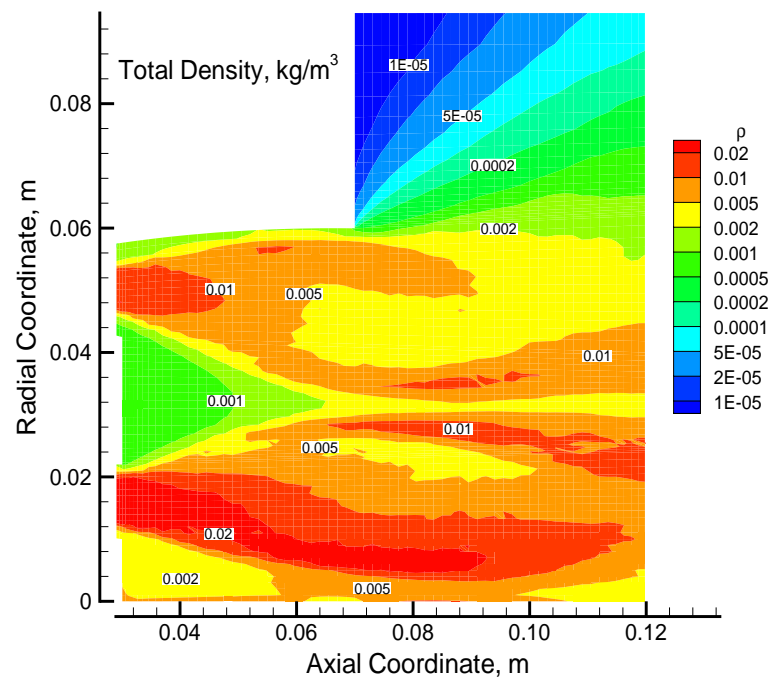

Figure 10. Total Density Field Predicted by the DSMC Simulation Using the Condensation Model

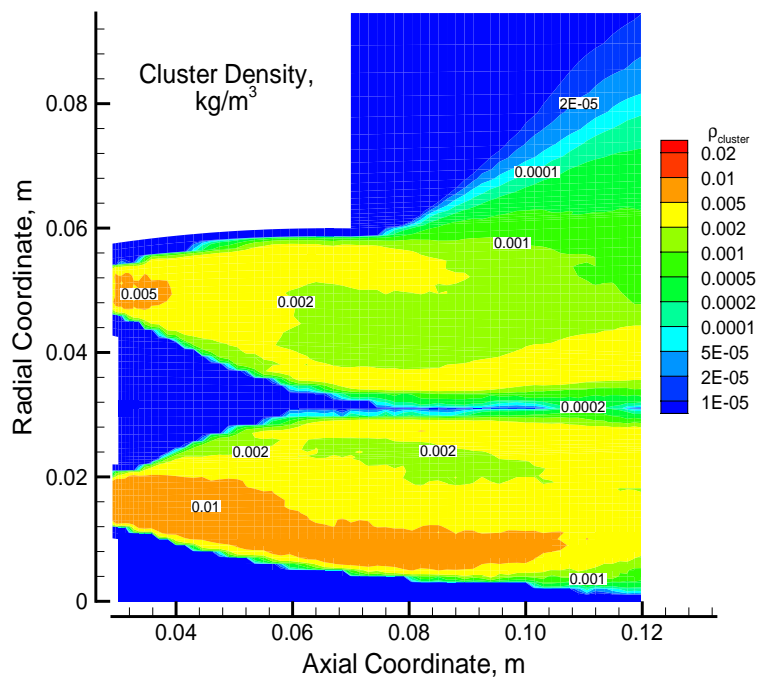

Figure 11. Cluster Mass Density Field Predicted by the DSMC Simulation Using the Condensation Model 
Effects of the amount of phase-change energy are also investigated. In general, the larger the magnitude of this energy, the wider is the expansion of the ring jet, and the higher is the temperature in the jets. Figures 13 and 14 compare the density distributions at axial locations of $0.072 \mathrm{~m}$ and $0.1 \mathrm{~m}$, respectively. The DSMC results using the condensation model are for when 30 percent of the latent heat of condensation is used as the phase-change energy. Clearly, the DSMC simulation is in better agreement with the data when the condensation model is used.

\section{Conclusions}

Simulating the Decade flow was a challenge for numerous reasons. First, the flow is very complex and thus requires significant computer resources to capture the flow structures.

Second, non-continuum nonequilibrium effects were important in some regions of the flow. The direct simulation Monte Carlo (DSMC) method was employed to simulate the flow in the low-density recess and vacuum chamber regions.

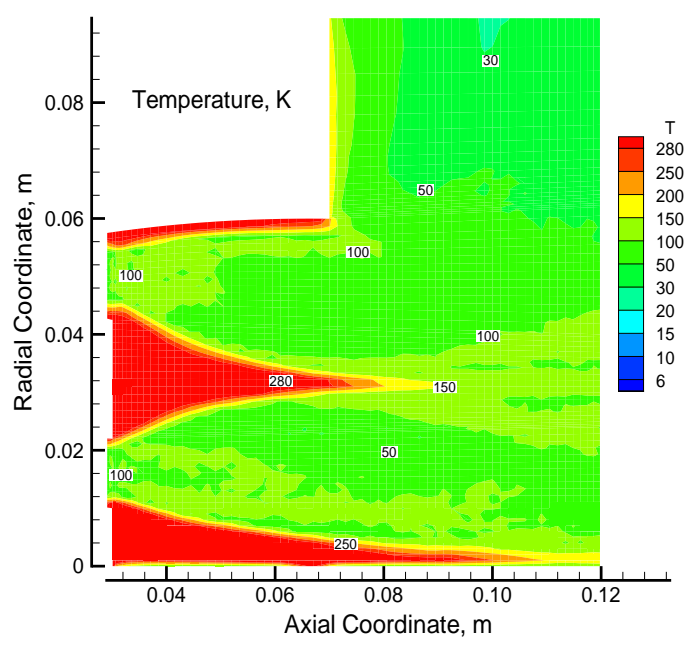

Figure 12. Temperature Field Predicted by the DSMC Simulation Using the Condensation Model

Third, the rapid gas expansion in the internal ring nozzles and recess regions results in very low temperatures, suggesting that homogeneous nucleation and condensation could occur in the Decade flow.

A homogeneous nucleation and condensation model was proposed and implemented in the DSMC method. The DSMC simulation using this model illustrated that the effects of gas condensation are significant. Reasonable agreement was obtained between simulation results and available experimental data, indicating the usefulness of the condensation model.

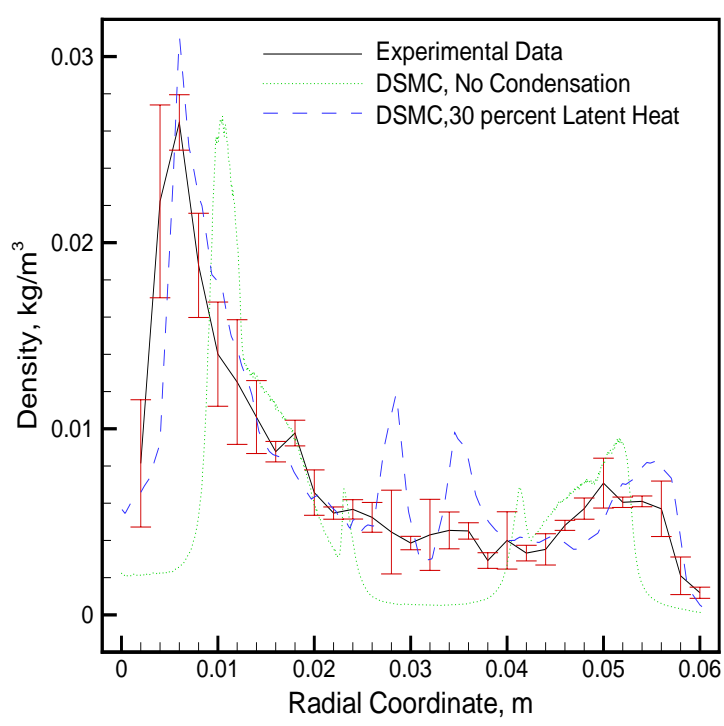

Figure 13. Comparison of Density Profiles at the Axial Location of $0.072 \mathrm{~m}$

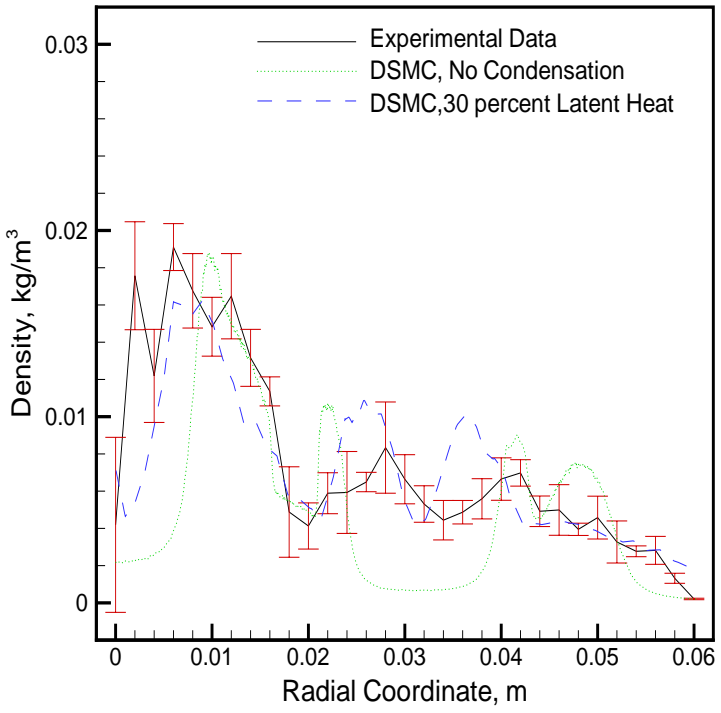

Figure 14. Comparison of Density Profiles at the Axial Location of $0.1 \mathrm{~m}$

\section{Acknowledgments}

This work was sponsored by the Air Force Office of Scientific Research, USAF, under AFOSR Grant F4962003-1-0123. The views and conclusions contained herein are those of the authors and should not be interpreted as necessary representing the official policies or endorsements, either expressed or implied, of the Air Force Office of Scientific Research or the U.S. Government. 


\section{References}

${ }^{1}$ Bird, G. A., Molecular Gas Dynamics and the Direct Simulation of Gas Flows, Oxford University Press, 1994.

${ }^{2}$ Abraham, F. F., Homogeneous Nucleation Theory, Academic Press, 1974.

${ }^{3}$ McDonald, J. E., "Homogeneous Nucleation of Vapor Condensation I. Thermodynamic Aspects," American Journal of Physics, Vol. 30, No. 12, 1962, pp. 870-877.

${ }^{4}$ McDonald, J. E., "Homogeneous Nucleation of Vapor Condensation II. Kinetic Aspects," American Journal of Physics, Vol. 31, No. 1, 1963, pp. 31-41.

${ }^{5}$ Oxtoby, D. W., "Homogeneous Nucleation: Theory and Experiment,” Journal of Physics: Condensed Matter, Vol. 4, 1992, pp. 7627-7650.

${ }^{6}$ Hettema, H., and McFeaters, J. S., "The Direct Monte Carlo Method Applied to the Homogeneous Nucleation Problem," Journal of Chemical Physics, Vol. 105, No. 7, 1996, pp. 2816-2827.

${ }^{7}$ Schenter, G. K., Kathmann, S. M., and Garrett, B. C. "Dynamical Nucleation Theory: A New Molecular Approach to Vapor-Liquid Nucleation,” Physical Review Letters, Vol. 82, No. 17, 1999, pp. 3484-3487.

${ }^{8}$ Toxvaerd, S., "Molecular Dynamics Simulations of Nucleation," Molecular Simulation, Vol. 30, No. 2-3, 2004, pp. 179182.

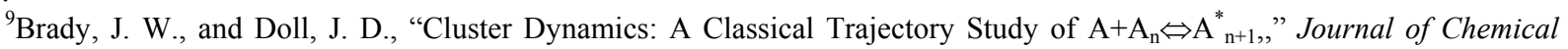
Physics, Vol. 71, No. 6, 1979, pp. 2467-2472.

${ }^{10}$ Hagena, O. F., and Obert, W., "Cluster Formation in Expanding Supersonic Jets: Effect of Pressure, Temperature, Nozzle Size, and Test Gas," Journal of Chemical Physics, Vol. 56, No. 5, 1972, pp. 1793-1802.

${ }^{11}$ Hill, P.G., "Condensation of Water Vapour during Supersonic Expansion in Nozzles," Journal of Fluid Mechanics, Vol. 25, No. 3, 1966, pp. 593-620.

${ }^{12}$ Hillenkamp, M., Keinan, S., and Even, U., "Condensation Limited Cooling in Supersonic Expansions," Journal of Chemical Physics, Vol. 118, No. 19, 2003, pp. 8699-8705.

${ }^{13}$ Zhong J., Gimelshein S. F., Zeifman, M. I., and Levin, D. A., "Modeling of Homogeneous Condensation in Supersonic Plumes with the DSMC Method," AIAA 2004-0166, 2004.

${ }^{14}$ Perrell, E. R., Erickson, W. D., and Candler, G. V., "Numerical Simulation of Nonequilibrium Condensation in a Hypersonic Wind Tunnel," Journal of Thermophysics and Heat Transfer, Vol. 10, No. 2, 1996, pp. 277-283.

${ }^{15}$ The General Aerodynamic Simulation Program, GASP Version 4.1, Computational Flow Analysis Software for the Scientist and Engineer, User's Manual, Aerosoft, Inc., Blacksburg, Virginia.

${ }^{16}$ Wang, W.-L., and Boyd, I. D., "Predicting Continuum Breakdown in Hypersonic Viscous Flows," Physics of Fluids, Vol. 15, 2003, pp. 91-100.

${ }^{17}$ Dietrich, S., and Boyd, I. D., "Scalar and Parallel Optimized Implementation of the Direct Simulation Monte Carlo Method," Journal of Computational Physics, Vol. 126, 1996, pp. 328-342.

${ }^{18}$ Hale, B.N., "Monte Carlo Calculations of Effective Surface Tension for Small Clusters," Austrian Journal of Physics, Vol. 49, 1996, pp. 425-434. 